\title{
Three-dimensional Induction Mapping of Magnetic Nanoscale Materials by Electron Holographic Tomography
}

\author{
Daniel Wolf ${ }^{1}$, Axel Lubk ${ }^{1}$ and Hannes Lichte ${ }^{1}$ \\ 1. Triebenberg Laboratory, Institute of Structure Physics, Technische Universität Dresden, Dresden, \\ Germany
}

Off-axis electron holography $(\mathrm{EH})$ is a powerful TEM technique that provides access to the 2D projections of both the electric potential, i.e., mainly the mean inner potential (MIP) contribution, and the components of the magnetic induction (B-field) that lie perpendicular to the electron beam path. Currently, 2D magnetic induction maps may be obtained with a spatial resolution of a few nanometer $[1,2]$ and a sensitivity of a few millitesla [2]. Frequently, however, nanomagnetic applications, such as spin valves or magnetic nanowires require a characterization of the 3D magnetic induction distribution. In the following, we therefore elaborate on how electron holography is combined with electron tomography to electron holographic tomography (EHT) [1,3] in order to retrieve the 3D distribution of the magnetic induction.

As shown in Fig. 1, the tomographic reconstruction of one B-field component starts with acquiring a tilt series of electron holograms that is subsequently reconstructed to obtain a tilt series of phase images. In order to collect data for the crucial separation of electric and magnetic phase shifts, either a tilt series over $360^{\circ}$ (Fig. 1), or two tilt series, one before and one after reversing the direction of magnetization in the specimen (e.g., using the TEM objective lens or flipping the sample up-side down in the holder), have to be acquired. Before separation of electric/magnetic phase shifts by computing half of the sum/difference of corresponding image pairs, relative displacements, rotations, and direction dependent magnification changes between the two phase images are corrected. Also, the alignment of the whole tilt series, i.e., an accurate tilt axis determination and subpixel displacements correction, is of crucial importance. Finally, from the aligned tilt series, tomograms of both the electric potential and the B-field component parallel to the tilt axis are reconstructed with tomographic reconstruction algorithms.

Fig. 2 shows two recent EHT studies revealing the B-field within a Co nanowire (NW) [4] and a $\mathrm{Co}_{2} \mathrm{FeGa}$ Heusler alloy NW [5] both with spatial resolution higher than $10 \mathrm{~nm}$. The reconstructions of the dominant axial component of the magnetic induction exhibit two interesting features for each case: At the Co NW, a small inversion domain at the apex was observed, whereas at the $\mathrm{Co}_{2} \mathrm{FeGa} \mathrm{NW}$, a magnetic dead layer of $10 \mathrm{~nm}$ width was found. These results were achieved by means of dedicated TEM sample holders for acquisition, in combination with advanced in-house developed software packages for acquisition, alignment and tomographic reconstruction. The powerful approach presented here is widely applicable to a broad range of 3D magnetic nanostructures and may trigger the progress of novel spintronic nonplanar nanodevices. In a next step the reconstruction of all B-field components is envisaged. [6]

References:

[1] P A Midgley and R E Dunin-Borkowski, Nat. Mater. 8 (2009) p. 271.

[2] M Körner et al, Small 10 (2014) p. 5161.

[3] D Wolf et al, Current Opinion in Solid State and Materials Science 17 (2013) p. 126. 
[4] D Wolf et al., Chem. Mater. 27 (2015) p. 6771.

[5] P Simon et al, Nano Letters 16 (2016) p. 114.

[6] This work was supported by the European Union under the Seventh Framework Program under a contract for an Integrated Infrastructure Initiative Reference 312483-ESTEEM2.

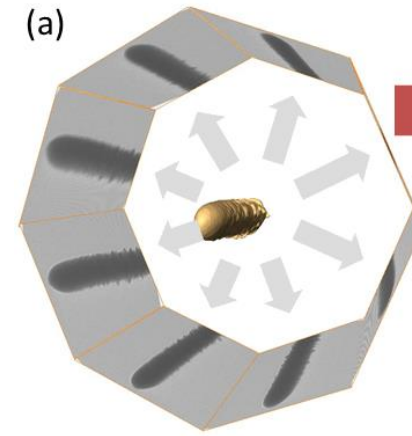

Hologram $360^{\circ}$ tilt series



Phase image $360^{\circ}$ tilt series (c)

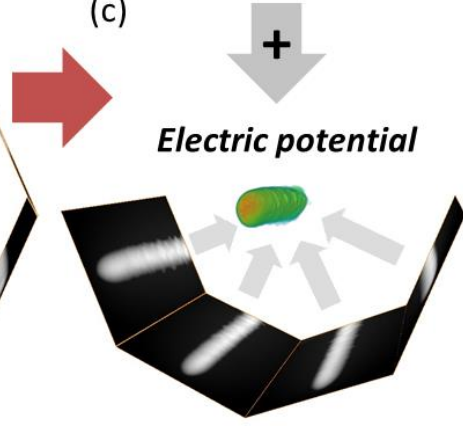

Electric phase image $180^{\circ}$ tilt series (d)

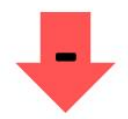

Magnetic field $B_{\text {axial }}$

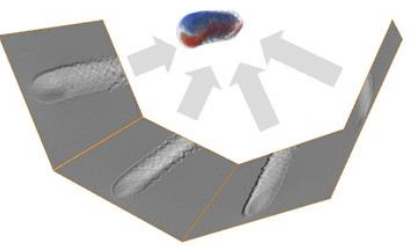

Magnetic phase derivatives $180^{\circ}$ tilt series

Figure 1. Principle of electron holographic tomography: (a) A tilt series of holograms covering a tilt range of $360^{\circ}$ is recorded. (b) From the holograms, the phase image tilt series is reconstructed and (c) separated in its electric/magnetic part by computing half of the sum/difference between opposite $\left(180^{\circ}\right.$ tilted) projections. The tilt series of electric phase images is used for tomographic reconstruction of the electric potential (c), whereas the partial derivatives of magnetic phase images perpendicular to the tilt axis are used for tomographic reconstruction of the B-field component parallel to the tilt axis (d).

\section{Cobalt Nanowire}

(a)

(c)

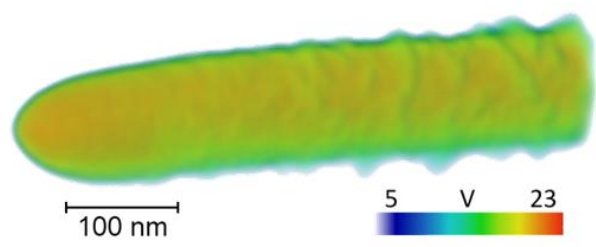

(e)


(b)

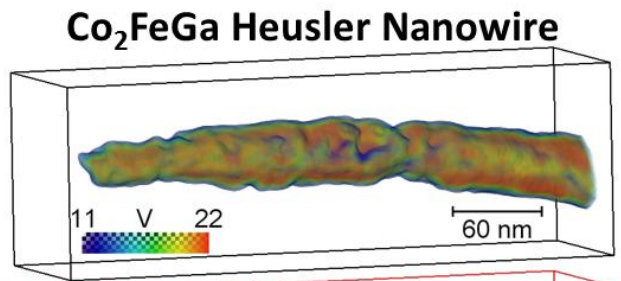

(d)

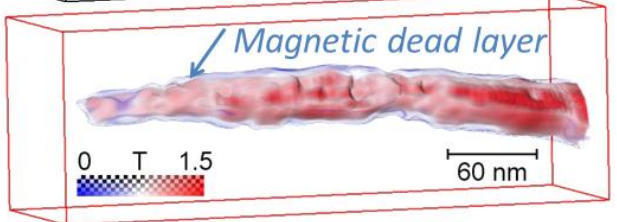

(f)



Figure 2. 3D reconstruction of magnetic nanowires (NWs). 3D Volume rendering of electric potential (a,b) and axial (predominant) B-field component (c,d) inside the NWs. Colors correspond to the potential/B-field values. (e) Axial B-field component inside the Co NW obtained from micromagnetic simulation. The arrow plots visualize the out-of-plane components showing the twist of magnetic induction. (f) Line scans in axial direction through the center of the $\mathrm{Co}_{2} \mathrm{FeGa} \mathrm{NW}$ from the tip to the back. This Figure is adapted from Refs. [4,5]. 